\title{
The $\beta$ - Amylase Gene Family in Brassica Napus: Genome Wide Analysis and Expression Profiles in Response to Abiotic Stresses
}

Yan Lv ( Ivyan322@163.com )

Key Laboratory of Biology and Genetic Improvement of Oil Crops, Ministry of 4 Agriculture, Oil Crops Research Institute of the Chinese Academy of Agricultural 5 Sciences, Wuhan 430062, China.

Dan Luo

Oil Crops Research Institute Chinese Academy of Agricultural Sciences

\section{Ziqi Jia}

Oil Crops Research Institute Chinese Academy of Agricultural Sciences

\section{Yong Cheng}

Oil Crops Research Institute Chinese Academy of Agricultural Sciences

\section{Xiling Zou}

Oil Crops Research Institute Chinese Academy of Agricultural Sciences

\section{Research article}

Keywords: Brassica crops, BAM gene family, Starch metabolism, Abiotic stresses

Posted Date: September 9th, 2020

DOI: https://doi.org/10.21203/rs.3.rs-70066/v1

License: (c) (i) This work is licensed under a Creative Commons Attribution 4.0 International License. Read Full License 


\section{Abstract}

Background: The $\beta$ amylase (BAM) gene family, known for their property of catalytic ability to hydrolyze starch to maltose units, has been recognized to play critical roles in metabolism and gene regulation. To date, BAM genes have not been characterized in oil crops.

Results: In this study, the genome wide survey revealed the identification of 30 BnaBAM genes in Brassica napus (B. napus), 11 BraBAM genes in Brassica rapa (B. rapa), 20 BoBAM genes in Brassica oleracea (B. oleracea), which were divided into 4 subfamilies according to the sequence similarity and phylogenetic relationships. All the BAM genes identified in the allotetraploid genome of $B$. napus, as well as two parental related species (B. rapa and B. oleracea), were analyzed for the gene structures, chromosomal distribution and collinearity, the sequence alignment of the core glucosyl hydrolase domains was further applied. 30 BnaBAMs, 11 BraBAMs and 17 BoBAMs exhibited uneven distribution on chromosomes of Brassica crops. The similar structural compositions of BAM genes in the same subfamily suggested that they were relatively conserved. Abiotic stresses pose one of the major constraints to plant growth and productivity worldwide. Thus, the responsiveness of BnaBAM genes under abiotic stresses were analyzed in B. napus. The expression patterns revealed a stress responsive behavior of all members, of which BnaBAM3s were more prominent. These differential expression patterns suggested an intricate regulation of BnaBAMs elicited by environmental stimuli.

Conclusion: Altogether, the present study provides first insights into the BAM gene family of Brassica crops, which lays the foundation for investigating the roles of stress-responsive BnaBAM candidates in B. napus.

\section{Full Text}

This preprint is available for download as a PDF.

\section{Figures}


Tree scale:

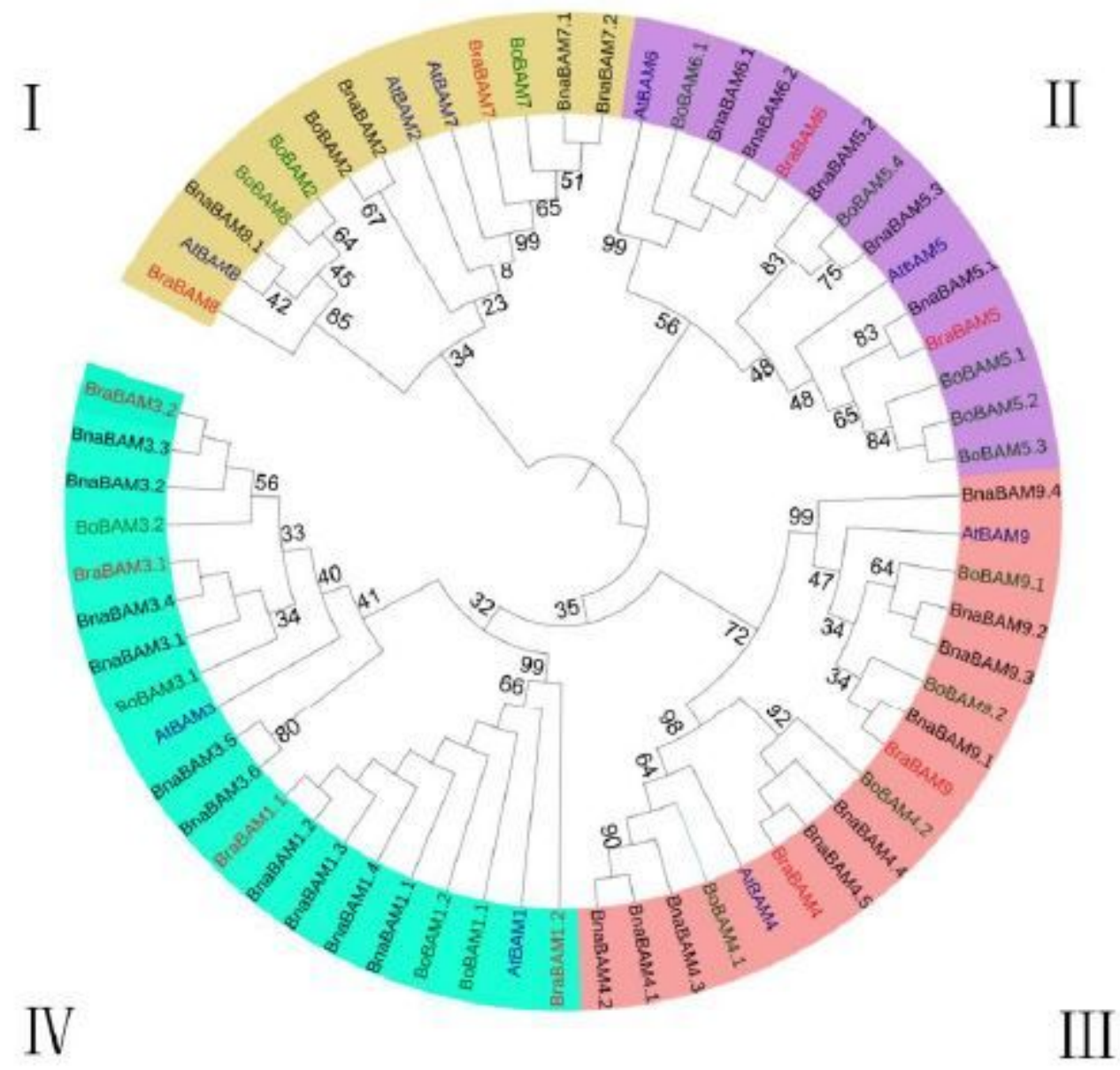

Figure 1

Phylogenetic analysis of BAM proteins in Arabidopsis, B. napus, B. rapa and B. oleracea. Bootstrap values are shown near the nodes. The different colored arcs represents different subfamilies of the BAM proteins. 


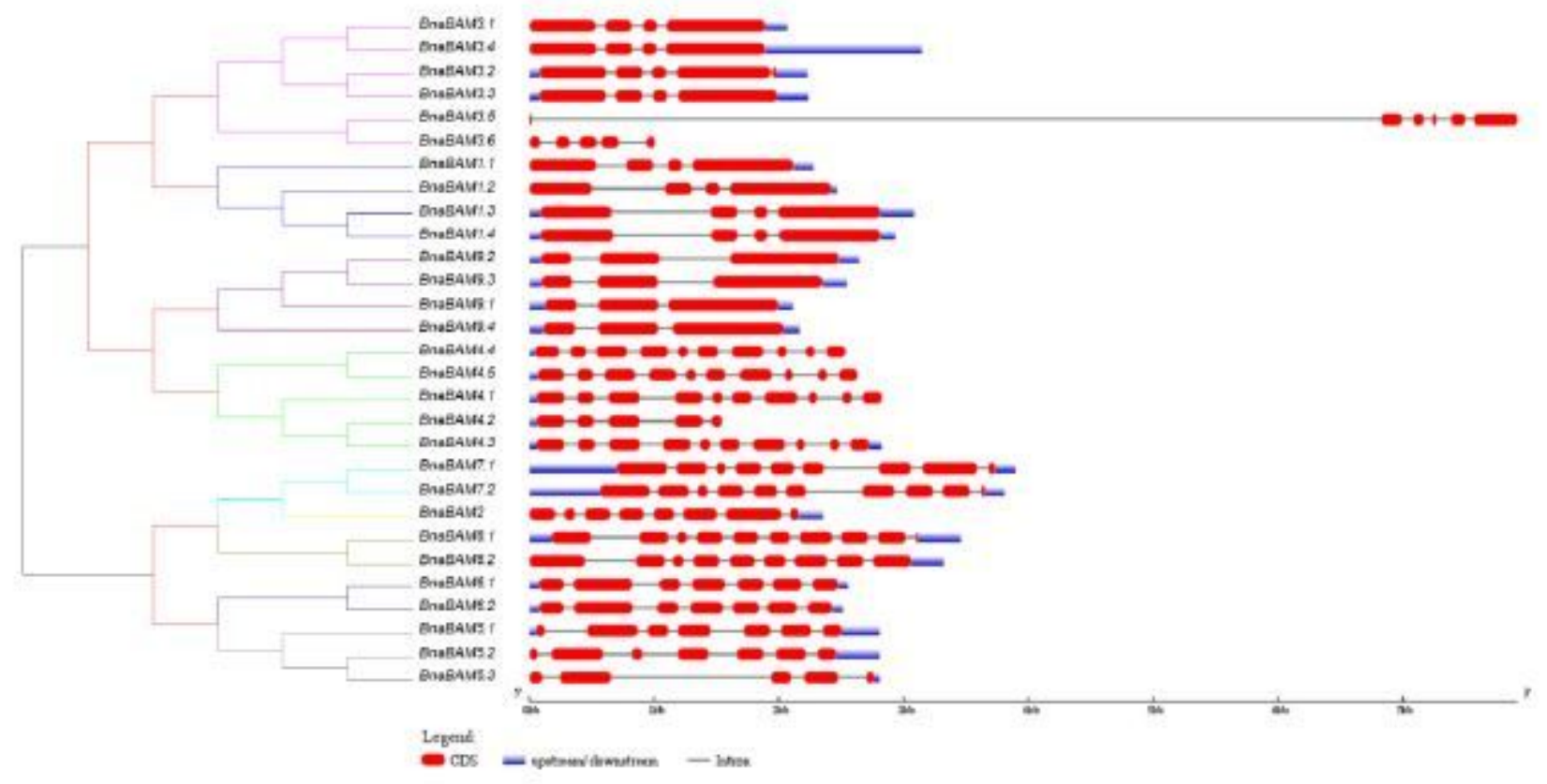

Figure 2

Gene structures of BnaBAM genes family. Gene structures of BnaBAMs based upon the number and position of exons (red boxes), introns (grey lines) and untranslated regions (blue lines). 

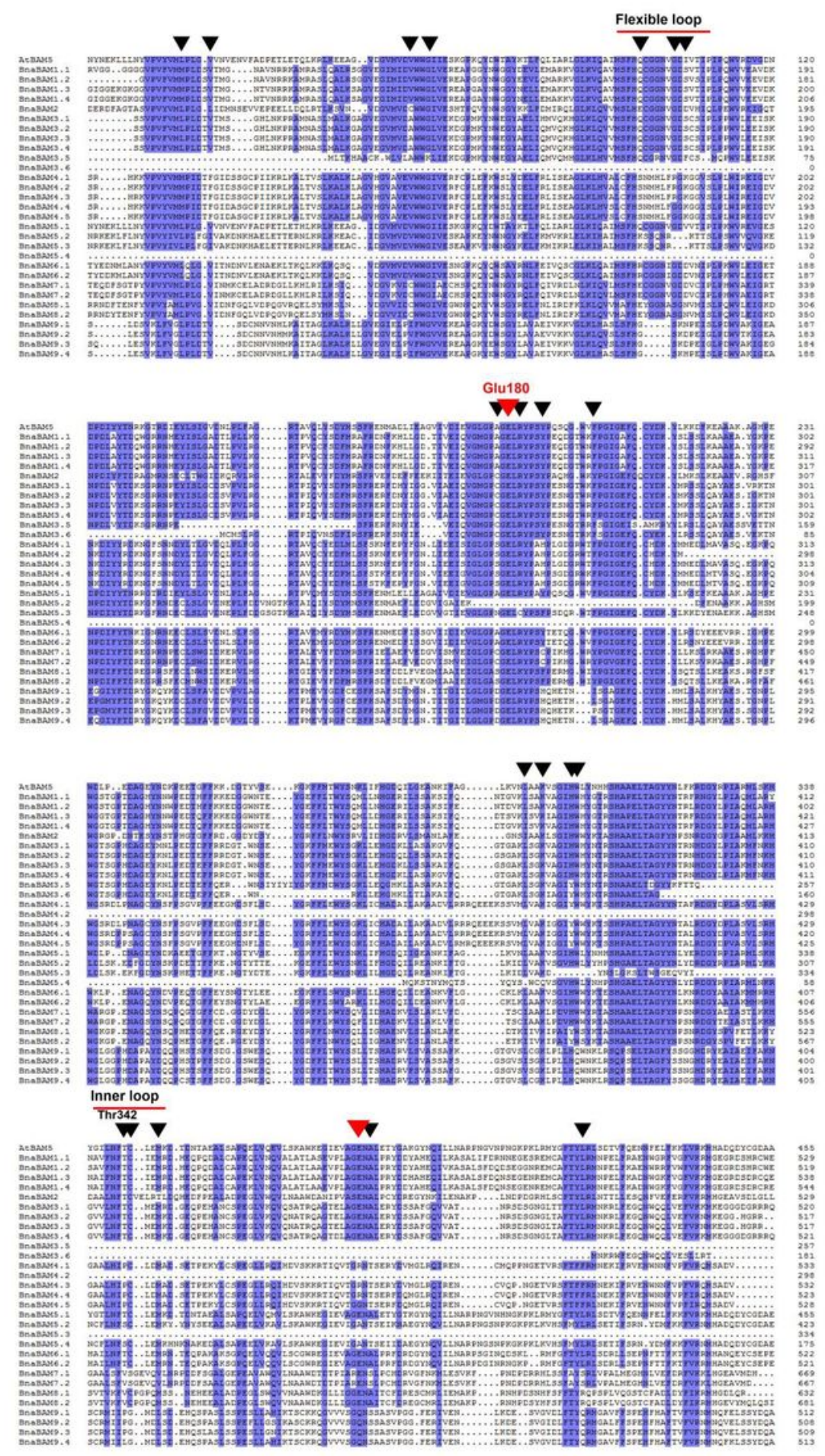

\section{Figure 3}

Alignment of the B. napus BAM proteins and the AtBAM5 protein of Arabidopsis. The blue shading indicate highly conservative substitutions. Unshaded residues are not conserved. Black arrowheads indicate substrate binding residues. Red arrowheads indicate the two catalytic residues. Red lines indicate the flexible loop structure. The alignment was made using ClustalX software program. 
A
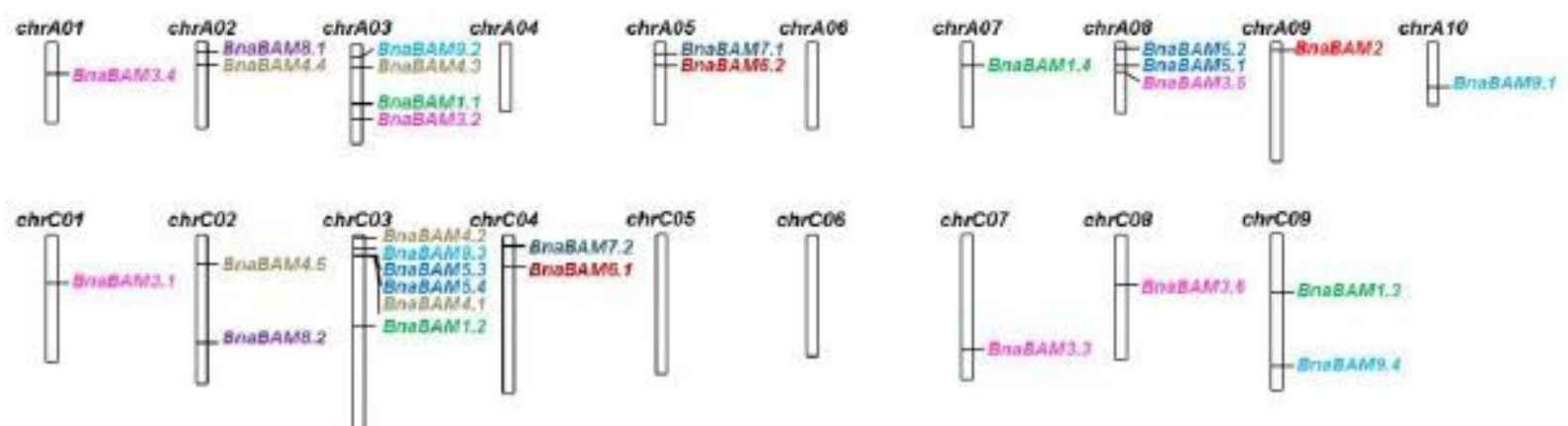

B
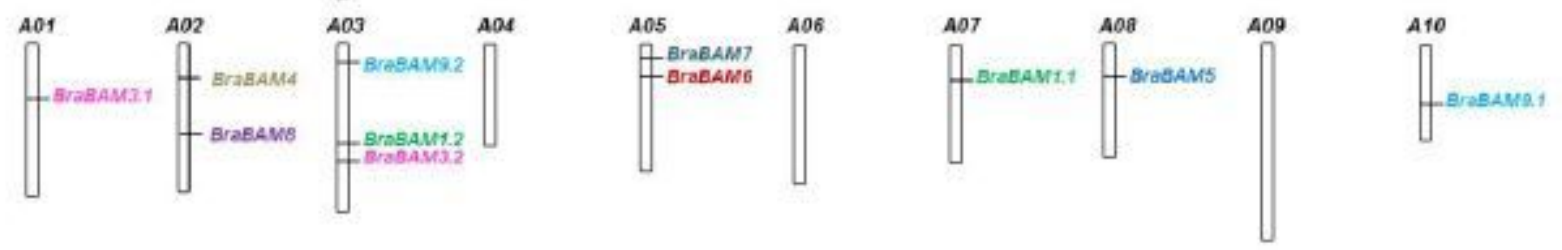

C
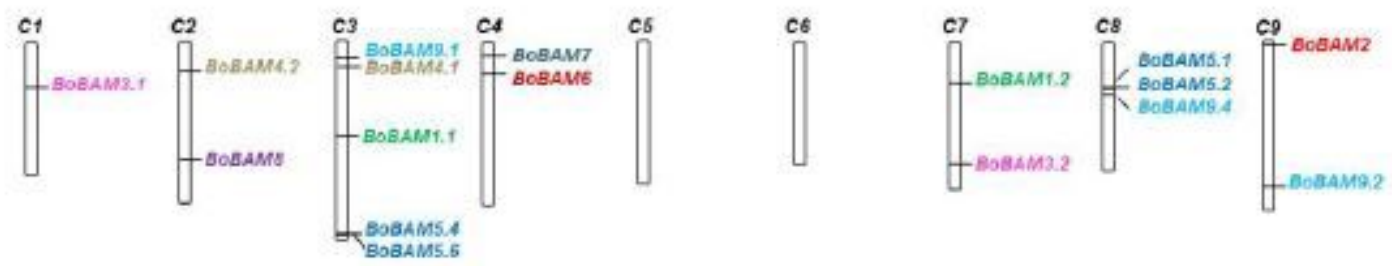

\section{Figure 4}

Chromosomal distribution of BAM gene family in B. napus, B. rapa and B. oleracea. The arrangement of 30 BnaBAM genes on 19 chromosomes of B. napus (A), 11 BraBAM genes on 10 chromosomes of $B$. rapa (B), and 17 BoBAM genes on 9 chromosomes of $B$. oleracea (C). 


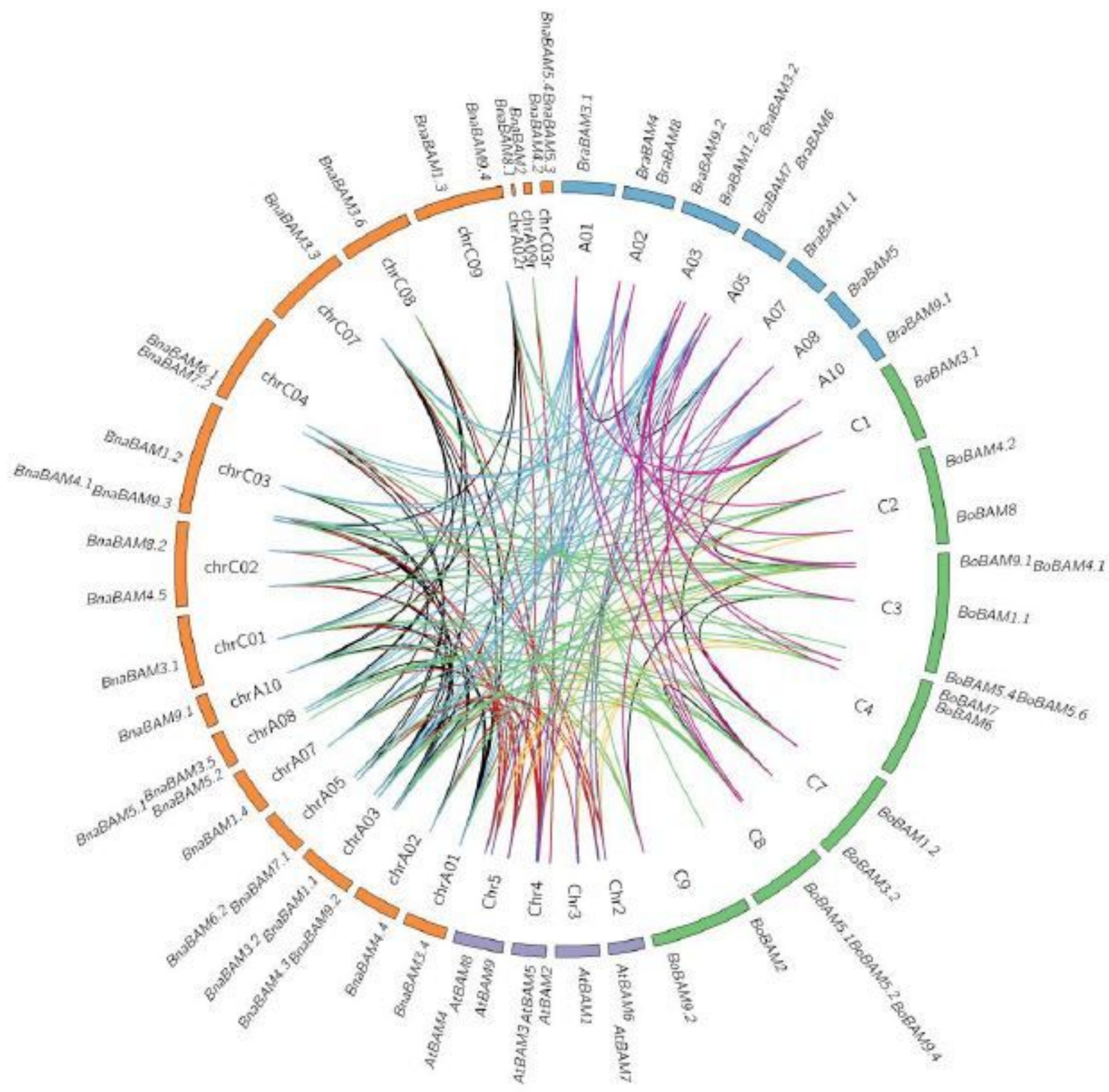

\section{Figure 5}

Collinearity analysis of the BAM genes between Arabidopsis and three Brassica 742 crops. The synteny relationship between each pair of BAM genes was detected using 743 linear regression. Genes with the syntenic relationship are linked by lines in different 744 color. The inner circle indicates the chromosome numbers and the outer circle 745 indicates the location of AtBAMs, BnaBAMs, BraBAMs and BoBAMs on 746 chromosomes of Arabidopsis, B. napus, B. rapa and B. oleracea. 

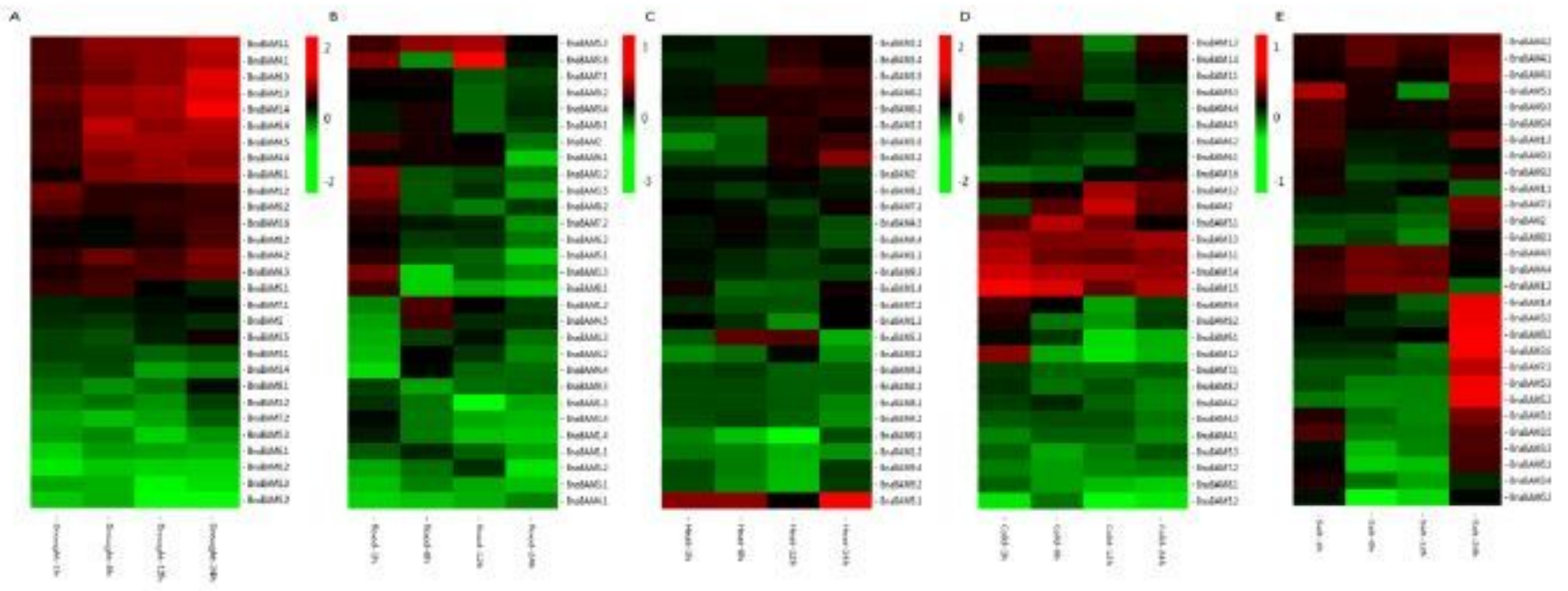

\section{Figure 6}

Expression profile of BnaBAM genes under different abiotic stresses conditions. 749 The transcripts of BnaBAM genes under drought (A), flooding (B), heat (C), cold (D) 750 and salt (E) conditions are investigated and depicted as heat maps. The color scale 751 represents log2(expression values), with red denoting high level transcription and 752 green denoting low level transcription.
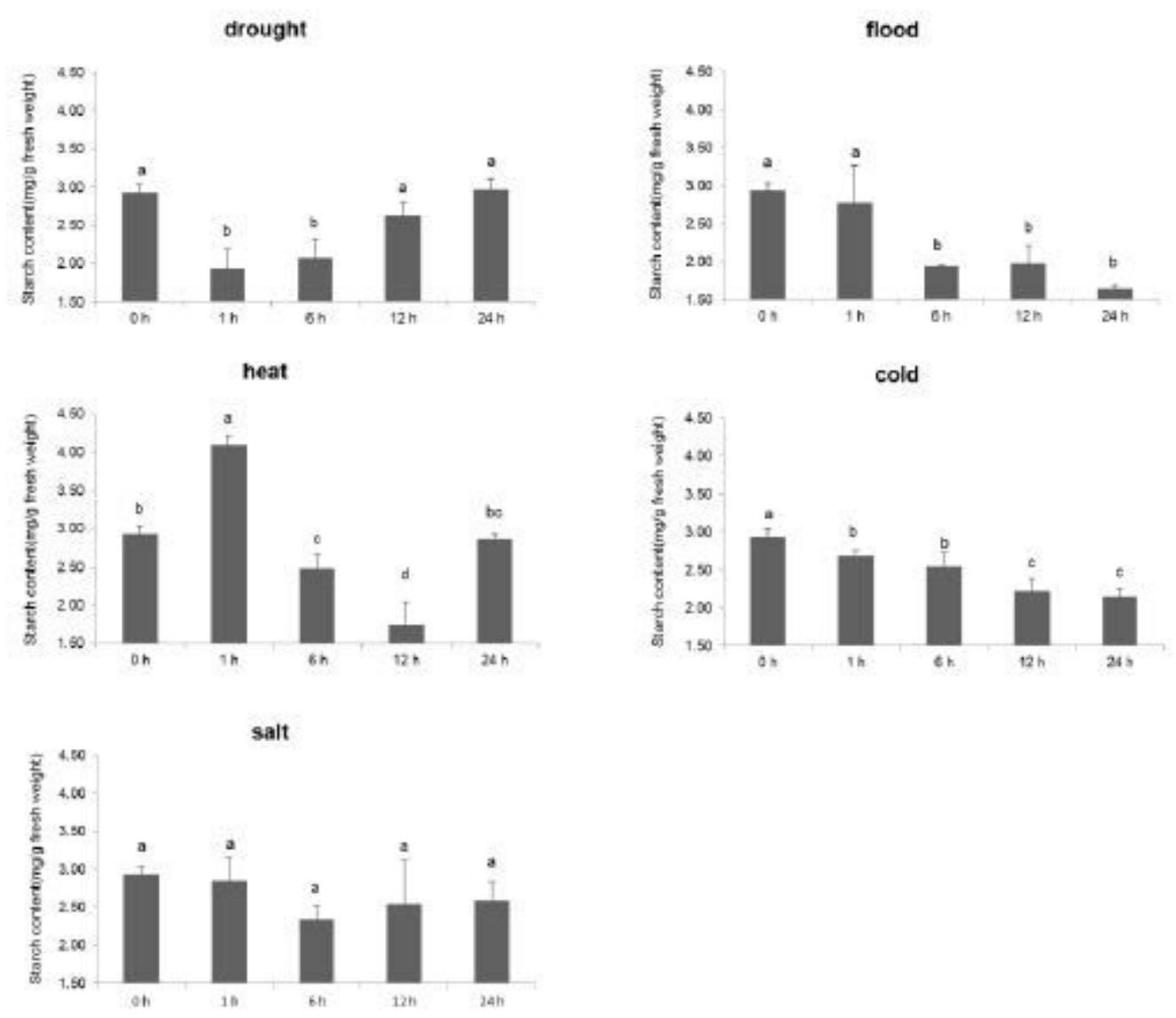
Figure 7

The effect of various abiotic stresses on starch content of B. napus. Investigations of starch content in B. napus seedling under drought (A), flooding (B), heat (C), cold (D) and salt (E) conditions. Bars indicate the SE of three biological replicates. Statistical analysis is determined by LSD test $(P<0.05)$
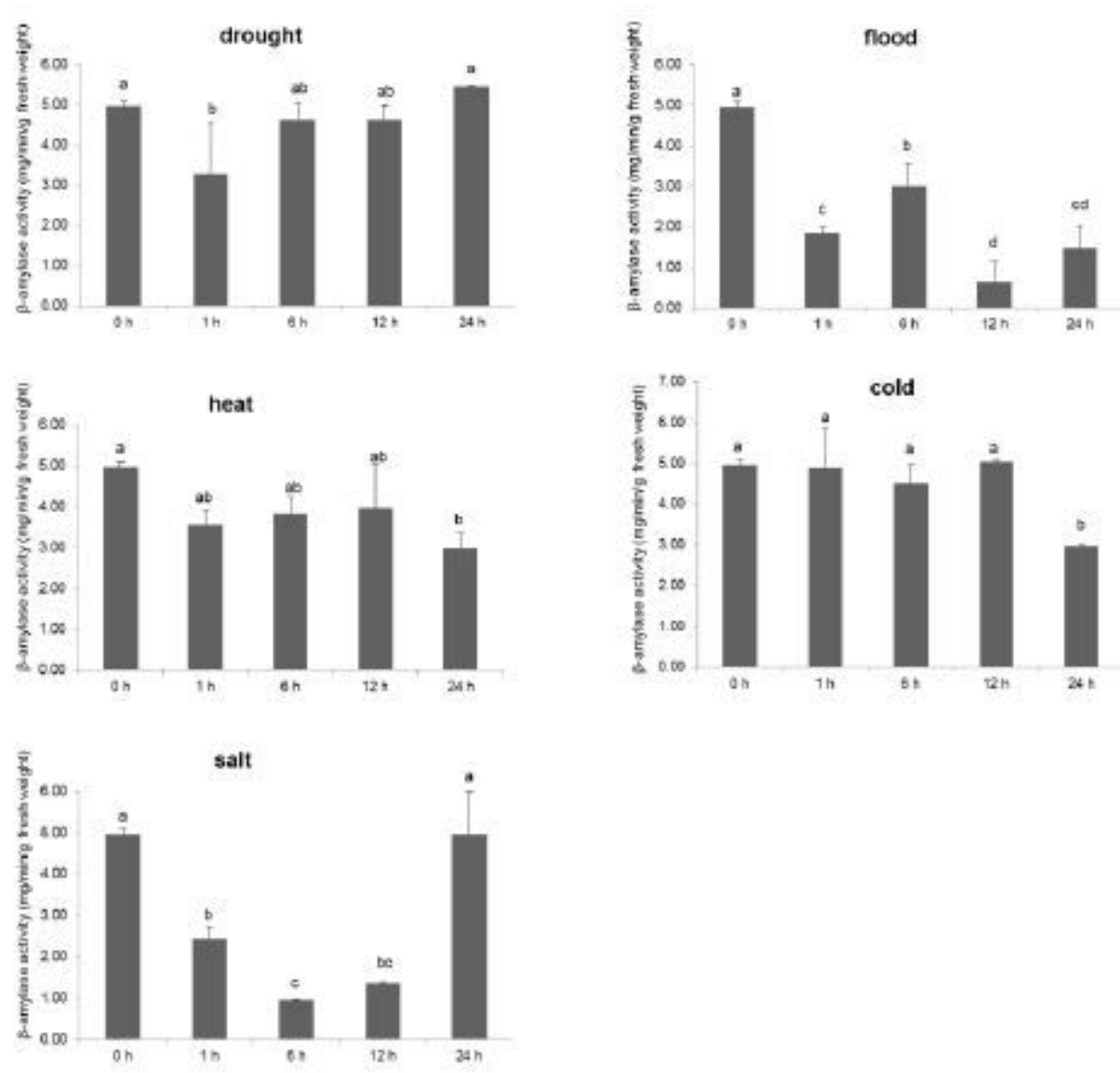

\section{Figure 8}

The effect of various abiotic stresses on $\beta$ amylase activity of $B$. napus. Investigations of $\beta$ amylase activity in $B$. napus seedling under drought (A), flooding (B), heat (C), cold (D) and salt (E) conditions. Bars indicate the SE of three biological replicates. Statistical analysis is determined by LSD test $(P<0.05)$.

\section{Supplementary Files}

This is a list of supplementary files associated with this preprint. Click to download.

- FigureS1.png

- TableS3.xIsx

- Tables2.xIsx 
- Tables1..xIsx

Page 10/10 